\title{
Comparative Study between Tramadol and Dexamethasone as an admixture to Bupivacaine in Supraclavicular Brachial Plexus Block.
}

\author{
Shrestha BR, ${ }^{1}$ Maharjan SK, ${ }^{1}$ Shrestha S, ${ }^{1}$ Gautam B, ${ }^{1}$ Thapa C, ${ }^{1}$ Thapa PB, ${ }^{2}$ Joshi MR ${ }^{2}$ \\ 'Department of Anaesthesiology, ${ }^{2}$ Department of Surgery, Kathmandu Medical College, Kathmandu, Nepal.
}

\begin{abstract}
This is a prospective, randomized, double blind study to evaluate the postoperative analgesia following supraclavicular brachial plexus block with Tramadol or Dexamethasone as an admixture to bupivacaine in upper extremity surgery.

Total 60 patients of ASA I and II undergoing upper extremity surgery under brachial plexus block with Bupivacaine were randomly divided in to two groups; one group received Tramadol $(2 \mathrm{mg} / \mathrm{kg})$ and the other group received Dexamethasone $(8 \mathrm{mg})$ as an admixture to Bupivacaine. The duration of postoperative analgesia was recorded in both groups using pain VAS score which was determined by maximum VAS score of 8-10 and when patient demands for additional analgesics.
\end{abstract}

The mean duration of postoperative analgesia in the Dexamethasone group was 1028.00 minutes while in the tramadol group it was 453.17 minutes

We concluded that Dexamethasone with local anaesthetic prolongs postoperative analgesia significantly than Tramadol $(\mathrm{P}<0.05)$ when used as admixture to local anaesthetic in brachial plexus block in upper extremity surgery.

Key words: Analgesia, Brachial plexus, Dexamethasone, Tramadol.

\section{INTRODUCTION}

Brachial plexus block is a popular and widely employed regional nerve block technique for perioperative anesthesia and analgesia for surgery of the upper extremity. Regional nerve block avoids the unwanted effect of the anaesthetic drugs used during general anesthesia and the stress of laryngoscopy and tracheal intubation. Minimizing the stress response and using minimal anesthetic drugs is always beneficial for the patients with various cardio respiratory comorbidities.

Nowadays different drugs have been used as adjuvants with local anesthetics in brachial plexus block to achieve quick, dense and prolonged block. ${ }^{1}$ Drugs like Morphine, Pethidine, Clonidine, Dexmeditomidine, Butorphanol, Midazolam are commonly used along with local anesthetics for this purpose. Since Morphine, Pethidine, Butorphanol are associated with side effects like heavy sedation, respiratory depression and psychomimmetic effects, drugs with minimal side effects are always looked for. Tramadol and Dexamethasone are selected as adjuvants to local anesthetics in brachial plexus block in this study because respiratory depression is not a major problem with their use, tramadol has been used as an adjunct to local anesthetic in axillary brachial plexus block and also

\section{Correspondence:}

Dr. Babu Raja Shrestha

Department of Anesthesiology \& Intensive Care Kathmandu Medical College, Kathmandu, Nepal. Email: barashrestha@yahoo.co.in 
in caudal anesthesia to extend the duration of postoperative analgesia. ${ }^{2,3}$

Steroids have nerve block prolonging effects. They block the nociceptive impulse transmission along the myelinated C fibres. ${ }^{4,5}$

Tramadol is an analgesic with mixed $\mu$ opioid and non opioid activity. It inhibits the reuptake of nor-epinephrine (NE) and serotonin from the nerve endings and potentiates the effect of local anesthetics when mixed together in peripheral regional nerve block. It has less respiratory depressant effect due to weak $\mu$ receptor affinity. ${ }^{5}$

\section{MATERIAL AND METHODS}

Main objective of this study was to determine whether Bupivacaine in combination with Tramadol or Dexamethasone increases the duration of analgesia following supraclavicular brachial plexus block in upper extremity surgery.

This is a prospective, randomized, double blind study of 60 patients (30 patients in each group) of American Society of Anaesthesiologists (ASA) physical grade I- II, aged between 18- 65 years, undergoing upper extremity surgery (elbow, forearm and hand) under supraclavicular brachial plexus block at Kathmandu Medical College (KMC), Kathmandu, Nepal. Approval for the study was taken from KMC ethical committee and written; informed consent was taken from the patients.

Exclusion criteria were ASA III-IV, Patient with coagulopathy, history of allergy to local anaesthetic, tramadol or dexamethasone, patchy or inadequate anesthesia, glucose intolerance, diabetes and acid peptic disease, requiring conversion to GA or when additional opioids or sedation administered, patient refusal and if develops subsequent pneumothorax.

Pre-anesthetic check up of the patients were done, premedicated with Diazepam $(0.1-0.2 \mathrm{mg} / \mathrm{kg})$ per operative in the night before and in the following morning on the day of surgery.

Patients were randomly allocated by the means of lottery to one of the two groups of 30 patients each. Group A received $0.5 \%$ Bupivacaine $2 \mathrm{mg} / \mathrm{kg}$ with Tramadol 2 $\mathrm{mg} / \mathrm{kg}$ and group B received $0.5 \%$ Bupivacaine $2 \mathrm{mg} / \mathrm{kg}$ with Dexamethasone $8 \mathrm{mg}$.

One of the investigator (unaware of the study group) prepared the solution in the syringe, specific code was given to the solution and at the end of the study decoding of the solution was done to respective study group. The solution was diluted with $15 \mathrm{ml}$ of distilled water. The brachial plexus on the side of surgery was blocked with the supraclavicular approach.

The supraclavicular block was performed in the supine position with the head turned to the opposite side and the arm placed medially toward the body. About $1.5 \mathrm{~cm}$ above the mid-clavicular point, a 22 G, 1.5 inches short beveled needle was introduced and directed just lateral to subclavian artery pulsation in caudad backward and medial direction. After hitting the first rib, paresthesia was elicited over the hand by walking the needle along the first rib. Keeping the needle in the same position, solution was injected slowly and ruling out intravascular injection intermittently. This was done by frequent aspiration through the syringe in between. The intercostobrachial nerve (T2) was blocked with $0.15 \%$ Bupivacaine $10 \mathrm{ml}$ on the upper middle aspect of the arm to avoid tourniquet pain in patients if planned for torniquet application.

The time of block performed was noted. Patients were monitored with electrocardiogram (ECG), pulse oxymetry $\left(\mathrm{SPO}_{2}\right)$, noninvasive blood pressure (NIBP) and respiratory rate.

The onset of sensory block was assessed every two minutes with application of cold spirit swabs and by response to atraumatic prick with the blunt needle in different areas innervated by radial, ulnar, median, musculocutaneous and antebrachial cutaneous nerves. The time when complete sensory blockade achieved was noted. The motor block was assessed every two minutes by asking the patient to raise their hand or move their fingers. When the patient could not move the fingers or raise the hand, this was considered as the complete motor block and the time was noted.

The surgery was allowed to proceed when complete anesthesia was achieved. The duration of surgery in each case was noted.

Postoperative follow up was carried out in the recovery and postoperative ward. The duration of analgesia was noted according to the 0-10 visual analogue scale (VAS) for pain at every hour till first 10 hours then 3 hourly till 24 hours.

VAS score 0 and 8-10 were taken as no pain and worst pain perceived, respectively. The duration of analgesia was determined till the existence of good analgesia. 
Shrestha et al. Comparative Study between Tramadol and Dexamethasone as an admixture to Bupivacaine...

When the patients began to experience the worst pain (VAS $=8-10$ ), it was considered that the analgesic action of the drugs was terminated. Patients were then rescued with additional analgesics on demand.

The duration of motor block postoperatively was assessed every two hourly by asking patient to move their fingers and to see whether they are able to raise the hand or not. This time was recorded and taken as cessation of motor block effect.

None of the patients were routinely sedated or given extra analgesics; until they started complain of pain. If any side effects related to the technique or drug such as nausea, vomiting, respiratory depression, pneumothorax occurred they were recorded and treated accordingly. The patient characteristics (age, sex, weight), duration of onset of sensory/motor block, duration of analgesia were recorded. The quantitative data was expressed in terms of mean (standard deviation) and comparison was done employing "independent sample t-test". P value was considered significant if it is less than 0.05 . Statistical calculation was done using Statistical Package for Social Science (SPSS) version 10.

\begin{tabular}{|lcc|}
\hline \multicolumn{3}{|l|}{ Table 1. Age Distribution } \\
\hline Characteristic & Tramadol* $^{*}$ & Dexamethasone* \\
Mean & 39.60 & 36.27 \\
Standard deviation & 13.36 & 15.01 \\
Minimum & 19 & 18 \\
Maximum & 68 & 65 \\
\hline
\end{tabular}

${ }^{*}$ Age in years

\section{RESULT}

The patient's characteristics (Table 1-3) in two study groups were similar. The age of the patient in Tramadol group ranged from 19 to 68 years and mean age was 39.60 years. In Dexamethasone group the age of the patient enrolled in this study ranged from 18 to 65 years. The mean age in this group was 36.27 years. The sex distributions in two groups are also similar. The mean body weight $(\mathrm{kg})$ in Tramadol and Dexamethasone were 58.17 and 56.40 respectively. The mean onset of motor block following the brachial plexus block in Tramadol group was 12.90 minutes and 13.43 minutes in Dexamethasone group. The difference of onset of motor block in two groups was not statistically significant $(P>0.05)$.

\begin{tabular}{|lcc|}
\hline \multicolumn{3}{|l|}{ Table 3. Body Weight Distribution } \\
\hline Characteristic & Tramadol* $^{*}$ & Dexamethasone* \\
Mean & 58.17 & 56.40 \\
Standard Deviation & 6.53 & 7.20 \\
Minimum & 45 & 42 \\
Maximum & 72 & 75 \\
\hline
\end{tabular}

*Weight in $\mathrm{Kg}$

The sensory block was achieved in mean 18.47 minutes in Tramadol group and 16.76 minutes in Dexamethasone group. The difference in onset of sensory block in two groups was statistically significant $(P<0.05)$.

The mean amount of Bupivacaine used in Tramadol groups was $115.33 \mathrm{mg}$ and $112.80 \mathrm{mg}$ in Dexamethasone group, which was not significant statistically $(P>.05)$.

The mean duration of surgery was 130.10 minutes in Tramadol group and 130.17 minutes in Dexamethasone group. Statistical test revealed the difference was not significant between two groups $(\mathrm{P}>.05)$.

The mean regression time for the motor block was 202.9 minutes in Tramadol group and 393.3 minutes in Dexamethasone group. The difference in duration of motor block in two group was significant statistically $(\mathrm{P}<.05)$.

\begin{tabular}{lcccc|}
\hline \multicolumn{2}{|l}{ Table 2. Sex Distribution } & & & \\
\hline Sex & Tramadol $^{*}$ & Dexamethasone* & Tramadol $^{\dagger}$ & Dexamethasone $^{\dagger}$ \\
Male & 16 & 19 & 53.3 & 63.3 \\
Female & 14 & 11 & 46.7 & 36.7 \\
Total & 30 & 30 & 100 & 100 \\
\hline * Frequency & & & & \\
$\dagger$ Percentage & & & \\
\hline
\end{tabular}


The mean duration of analgesia in Tramadol group was 453.17 minutes, whereas in Dexamethasone group it was 1028.17 minutes. This showed significant statistical difference $(P<.05)$ when compared to the duration of analgesia produced by two different drugs as adjuncts to local anesthetic in brachial plexus block.

The maximum VAS score for pain (8-10) in Tramadol group was recorded at 445-452 minutes postoperatively, whereas in dexamethasone group it was recorded after 1010-1015 minutes postoperatively.

\section{DISCUSSION}

Supraclavicular brachial plexus block is widely employed regional nerve block to provide anesthesia and analgesia for the upper extremity surgery. Supraclavicular block provide anesthesia of the entire upper extremity in the most consistent manner of any brachial plexus techniques. It is the most effective block for all portions of the upper extremity and is carried out at the "division" level of the brachial plexus, ${ }^{6}$ with high volume the trunk level of the plexus may also be blocked in this approach. Local anesthetics are used for this purpose. Currently available local anesthetics can provide analgesia for limited period of time when used as single injection. The nerve stimulator can be used to aid the location of the brachial plexus and plain bupivacaine used by this method has been claimed to produce the block as long as 3-8 hours. ${ }^{7}$ Practically the same result could not be produced in series of study with sole bupivacaine. To extend the analgesia period beyond the operation rooms, various methods have been tried with the aim of prolonging the local anesthetic action, like continuous infusion of local anesthetics via indwelling catheters, use of different additive in local anesthetics.

In this study Tramadol or Dexamethasone was used as adjuvant in local anesthetic. Steroids block the transmission of impulse in nociceptive $C$ fibres, ${ }^{4,10}$ thus when used with local anesthetics steroids prolong the duration of local anaesthetic block. Tramadol when used with local anesthetics inhibits the reuptake of serotonin from nerve endings and potentiate the block effects. ${ }^{2,3,5}$ Moreover these two drugs lack respiratory depressant effects unlike the opioids mentioned above.

In this study the patient characteristics (age, sex and weight) were similar in two groups. It has been found that the total duration of analgesia following the brachial plexus block in the steroid group was significantly higher than in Tramadol group. The Duration of analgesia with Tramadol in brachial plexus block in the study by Chattopadhya, Mitra et al was $410.1 \pm 95.1$ minutes. $^{8}$
Many previous workers have included Dexamethasone in Bupivacaine micro-spheres to see the block effect in animal models and found prolonged duration of block (7 -11 days) when steroid was used together. ${ }^{5}$ Additional of small amount of dexamethasone to bupivacaine microcapsules prolongs the local analgesia when compared to micro-capsules with plain bupivacaine after subcutaneous placement in humans., ${ }^{9,10,11,15}$

Kopacz DJ, Lacouture PG et al explored the effect of Dexamethasone in bupivacaine micro-capsule for intercostal blockade in healthy human volunteers and concluded that Dexamethasone micro-capsules are well tolerated and inclusion of Dexamethasone increased the duration of intercostal block to at least 96 hours. ${ }^{11}$ Methyl prednisolone added to local anaesthetic in axillary block produced the prolong nerve block in the study conducted by Stan ,Goodman et al. ${ }^{12}$

The onset of motor block was faster than the sensory block in either of the group in this study. As described by Winnie in 1977 the outer motor fibers are blocked earlier than the sensory fibers which are situated deeper in the brachial plexus at the level of trunk and division. ${ }^{13,17}$ Our study showed the same result that the motor block was quicker than the sensory block.

The regression time of the motor block in the postoperative period was studied. In Tramadol group the mean duration of return of the motor function was after 202.9 minutes and in Dexamethasone group it was 393 minutes after the block was performed. The difference in the regression time of the motor block was found to be statistically significant $(P<.05)$. Prolonged regression time may cause limitation of the neurological evaluation of the extremity in selected cases. de Jong et al studied the motor function returned after nerve block and found the motor function returns before pain perception. ${ }^{14,17}$ The anatomical location of surgery was classified into elbow, forearm, wrist and hand areas. Distributions of location of surgery in two study groups were similar (Table 4). The average duration and the invasiveness of the surgeries in the two groups were also similar.

\begin{tabular}{|lccc|}
\hline \multicolumn{4}{l}{ Table 4 . Anatomical sites of surgical intervention } \\
\hline Site & Tramadol & Dexamethasone & Total \\
Elbow & 11 & 12 & 23 \\
Forearm & 8 & 7 & 15 \\
Wrist & 8 & 7 & 15 \\
Hand & 3 & 4 & 7 \\
Total & 30 & 30 & 60 \\
\hline \hline
\end{tabular}


Shrestha et al. Comparative Study between Tramadol and Dexamethasone as an admixture to Bupivacaine...

Table 5. Comparative Study of Duration of Total Analgesia in Two Study Groups.

\begin{tabular}{|c|c|c|c|c|c|}
\hline Charateristic & Study Groups & Number & Mean & Standard Deviation & P-value \\
\hline \multirow{2}{*}{ Amount of drug in $\mathrm{mg}$} & Tramadol & 30 & 115.33 & 15.96 & \\
\hline & Dexamethasone & 30 & 6.49 & 0.92 & \\
\hline \multirow{2}{*}{ Total Bupivacaine (mg) } & Tramadol & 30 & 115.33 & 15.97 & \multirow{2}{*}{0.521} \\
\hline & Dexamethasone & 30 & 112.80 & 14.41 & \\
\hline \multirow{2}{*}{$\begin{array}{l}\text { Onset of motor block in } \\
\text { minutes }\end{array}$} & Tramadol & 30 & 13.93 & 1.66 & \multirow{2}{*}{0.196} \\
\hline & Dexamethasone & 30 & 12.90 & 1.49 & \\
\hline \multirow{2}{*}{$\begin{array}{l}\text { onset of sensory block } \\
\text { in minutes }\end{array}$} & Tramadol & 30 & 18.47 & 2.03 & \multirow{2}{*}{0.004} \\
\hline & Dexamethasone & 30 & 16.76 & 2.34 & \\
\hline \multirow{2}{*}{$\begin{array}{l}\text { Duration of surgery in } \\
\text { minutes }\end{array}$} & Tramadol & 30 & 130.10 & 46.89 & \multirow{2}{*}{0.996} \\
\hline & Dexamethasone & 30 & 130.17 & 52.74 & \\
\hline \multirow{2}{*}{$\begin{array}{l}\text { Regression of motor } \\
\text { Block in minutes }\end{array}$} & Tramadol & 30 & 202.93 & 30.55 & \multirow{2}{*}{0.000} \\
\hline & Dexamethasone & 30 & 393.03 & 98.96 & \\
\hline \multirow{2}{*}{$\begin{array}{l}\text { Total analgesia duration } \\
\text { in minutes }\end{array}$} & Tramadol & 30 & 453.17 & 72.81 & \multirow{2}{*}{0.000} \\
\hline & Dexamethasone & 30 & 1028.17 & 194.51 & \\
\hline \multirow{2}{*}{$\begin{array}{l}\text { Max. pain VAS Score } \\
(8-10)\end{array}$} & Tramadol & \multicolumn{3}{|c|}{ 435-450 minutes } & \\
\hline & Dexamethasone & \multicolumn{3}{|c|}{ 1010-1015 minutes } & 0.000 \\
\hline
\end{tabular}

Total duration of analgesia was determined by the intolerable pain (VAS, 8-10) and when patient demanded for analgesics. This was regarded as the end point for total analgesia period which was assessed hourly by the observer blinded to the study. Mild and moderate tolerable pain before pain VAS score 8 not needing rescue analgesics continued to contribute in VAS scoring till the end point. The difference of time duration postoperatively for the maximum VAS score in two groups was statistically significant. During the study three cases were withdrawn because of the block failure (patchy anesthesia) and converted to GA. The same number of the withdrawn cases with respective coding were replaced later and blinded to the observer.

Four cases in Tramadol group had nausea in the early postoperative period. Three cases had a single episode of nausea, whereas one case had nausea twice. The nausea was corrected on its own without any rescue antiemetics. None of the patients had respiratory complications in perioperative period. The follow up of patients for the duration of 1 to 6 months did not reveal any local complications (infection, neuritis) associated with the block in this study.
The addition of either of the test drugs to bupivacaine solution did not make the solution cloudy. In this study, the dose range of drug was determined according to the previous experiences of workers in different study $9,10,11$ and that was within the range of safe clinical dosage.

Tramadol is an analgesic with mixed $\mu$ opioid and non opioid activity. The non opioid activity is mediated via alpha-2 gonist mechanism and serotonin reuptake inhibition in the central nervous system. It inhibits the reuptake of nor-epinephrine and serotonin from the nerve endings and it is supposed to potentiate the effect of local anesthetics when mixed together. Robaux et al ${ }^{2}$ conducted a randomized double blinded clinical trial to assess the effect of Tramadol added to brachial plexus anesthesia in which 100 patients planned for carpal tunnel release surgery under brachial plexus anesthesia were randomized into different groups (group I, Tramadol 40 mg; group II, Tramadol $100 \mathrm{mg}$ and group III, Tramadol $200 \mathrm{mg}$ ). The number of patient requesting analgesia in postoperative period was significantly less in group III. This study demonstrate that Tramadol added to mepivacaine for brachial plexus block extends the duration and improves the quality of postoperative analgesia in a dose dependent fashion. ${ }^{2}$ 


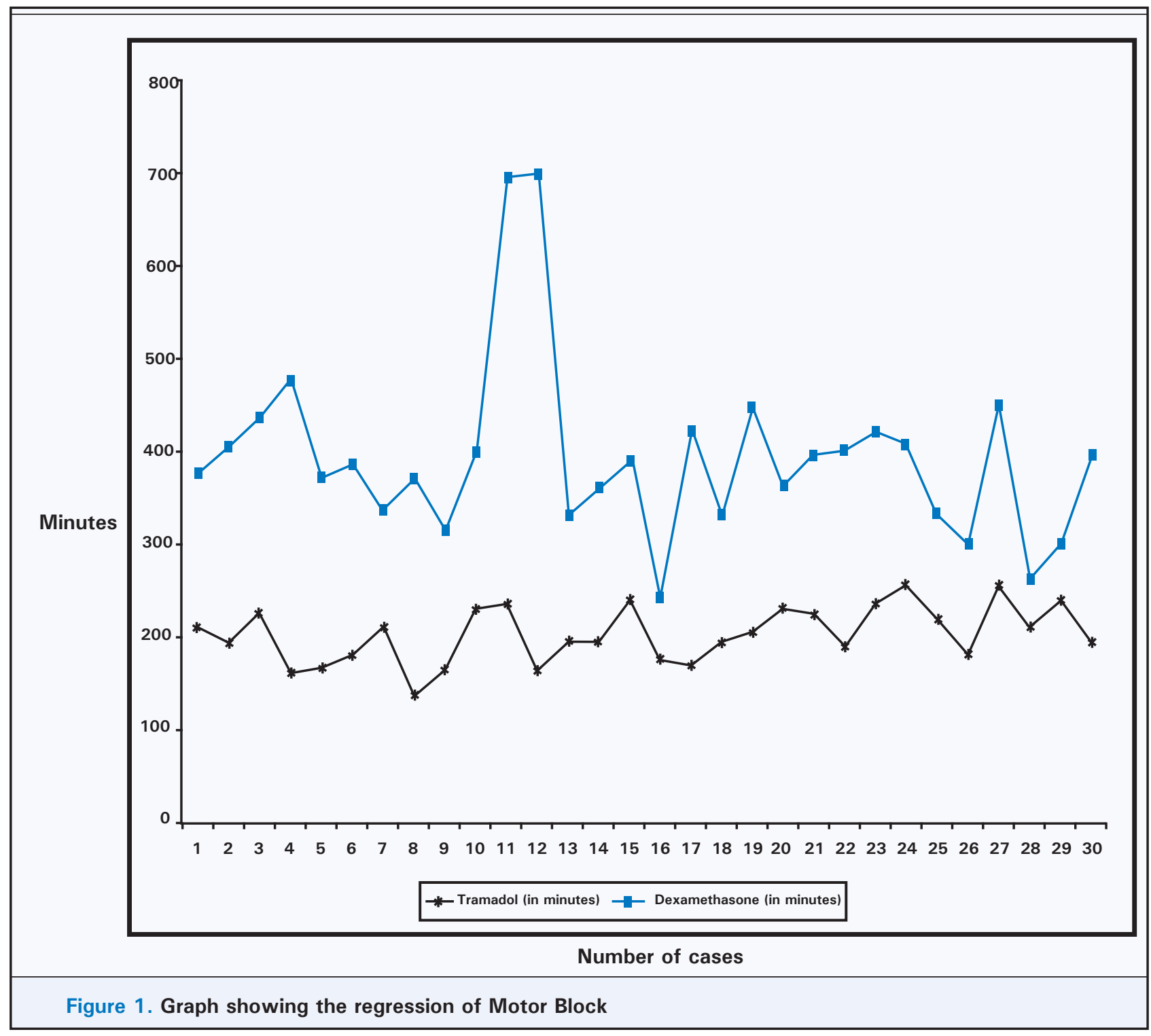

The location of the brachial plexus was done by paresthesia method using a 22 gauge short beveled 1.5 inches needle. The positive end point for paresthesia was taken when the patient complained of tingling sensation over different part of the extremity to be blocked and over as wide area as possible.

Steroids have block prolonging effect according to their anti-inflammatory potency, Dexamethasone prolongs the action of local anesthetics when used together. ${ }^{10}$ The pharmacodynamics and pharmacokinetics of the drugs when administered in regional nerve block is difficult to explain. There are some proposed mechanisms of actions of glucocoricoids when used with bupivacain micro-spheres to extend the block effect. ${ }^{15}$ The author assumes the similar probable mechanisms when corticosteroid was mixed with bupivacain solution, which is easily available and not pharmaceutically difficult to manufacture as micro-sphere formulations. The dense and earlier motor block in the steroid group is due to the synergestic action with local anaesthetic on blockade of nerve fibres. ${ }^{10}$

The block prolonging effect of dexamethasone is due to its local action and not a systemic one. ${ }^{16,17}$ It has been found that this effect of steroid is mediated via steroid receptors. ${ }^{8}$ When steroid alone was used to block the nerves the effect was not prolonged. ${ }^{10,14,16}$ The action of steroid has been related with the alteration of function of potassium channels on the excitable tissues..$^{5,14,16,18}$

\section{CONCLUSION}

Addition of Tramadol or Dexamethasone to local anesthetics in brachial plexus block prolonged postoperative analgesia. Dexamethasone when added 
to local anesthetic in brachial plexus block significantly prolonged postoperative analgesic duration (mean 1028 minutes) which was significantly higher in comparison with Tramadol as the adjuvant to local anaesthetic. This helps to minimize the cost and provides patient comfort. Such technique will be useful to be employed in our part of world where one has to face short of drugs and equipments while providing anesthetic services.

\section{REFERENCES}

1. Bazin JE, Massoni C, Bruelle P, Fenies V, Groslier D, Schoeffler $P$. The addition of opioids to local anaesthetics in brachial plexus block: the comparative effects of morphine, buprenorphine, and sufentanil. Anaesthesia 1997;52:858-62.

2. Robaux S, Blunt C, Viel E, et al. Tramadol added to $1.5 \%$ Mepivaciane for axillary brachial plexus block improves postoperative analgesia. Anaesth Analg 2004 April;98(4):1172-7.

3. Kapral S, Gool ann G, walt B, et al. Tramadol added to mepivacine prolongs the duration of an axillary brachial plexus blockade. Anaesth Analg 1999;88:853-66.

4. P. Prithvi Raj. Pain medicine a comprehensive review. New York: Mosby publication; 2005.

5. Evers AS, Maze M. Anesthetic pharmacology: Physiologic Principles and clinical practice. A companion to Millers Anaesthesia. Philadelphia: Joan Sinclair; 2004.

6. Grogono AW, Moudgil G. 6th Annual New Orleans Anaesthesiology Comprehensive Review and Update. New Orleans: 1977.

7. Lund PC, Cwik JC, Vallesteros F. Bupivacaine - a new long acting local anaesthetic agent. A preliminary clinical and laboratory report. Anaesth Analg 1976;49:103-13.

8. Chattopadhyay S, Mitra LG. Tramadol as an adjuvant for Brachial plexus block. J Anaesth Clin Pharmacol 2007;23(2):1879.

9. Castillo Jenny, Curley J, Hotz J, et al. Glucocorticoids prolong rat sciatic nerve blockade in vivo from bupivacaine microspheres. Anaesthesiology 1996;85:1157-66.

10. Johanson A, Hao J. Local corticosteroid application blocks transmission in normal nociceptive c-fibres. Acta Anesthesiologica scand 1990;34:335-8.
11. Kopacz DJ, Lacouture PG, Wu D, et al. The dose response and effects of dexamethasone on bupivacine microcapsules for intercostals blockade in healthy volunteers. Anaest Analg 2003;96:576-82.

12. Stan T, Goodman E, Cardida B, Curtis RH. Adding methylprednisolone to local anaesthetic increases the duration of axillary brachial plexus block. Reg Anaesth Pain Med 2004;29:380-1.

13. Winnie AP, Tay CH, Patel KP, Ramamurthy S, Durrani Z. Pharmacokinetics of local anaesthetics during plexus blocks. Anaesth Analg 1977;56:852-61.

14. de Jong RH; Wagman IH. Physiological mechanism of peripheral nerve block by local anaesthetics. Anaesthesiology 1963;24:684-7.

15. Effects of dexamethasone on motor brachial plexus block with bupivacaine loaded microspheres in a sheep model. Eur J Anesthesiol 2003;20(4):305-10.

16. Marks R, Barlow JW, Funder JW. Steroid induced vasoconstriction: glucocorticoid antagonist studies. J Clin Endo Meta 1982;54:1057-7.

17. Koj Jarbo, Yatindra KB, Nidhi BP. Brachial plexus block with midazolam and bupivacaine improves analgesia. Can J Anaesth 2005;52(8):822-6.

18. Castillo J, Curley J, Hotz J, et al. Glucocorticoids prolong rat sciatic nerve blockade in vivo from bupivacaine microspheres. Anesthesiology 1996;85:1157-66.

19. Partridge BL, Katz J, Bernirschke K. Functional Anatomy of brachial plexus sheath. Implications for Anaesthesia. Anaesthesiology 1987;66:743-7. 